\title{
Inward continuation of the scalp potential distribution by means of the vector BEM
}

\author{
M.J. van Burik, M.C. Mulder, J.G. Stinstra, M.J. Peters \\ Institute for Biomedical Technology (BMTI), Low Temperature Group, Dept. of Applied Physics, \\ University of Twente, P.O. box 217, 7500 AE Enschede, the Netherlands, burik@dpmi.tu-graz.ac.at
}

\begin{abstract}
The vector Boundary Element Method (vBEM) is used for the calculation of a matrix that links the tangential components of the current density on the cortical and scalp surface. This so-called transfer matrix is compared to the transfer matrix that links the potential distribution on both surfaces. Forward and inverse calculations are performed to evaluate both types of transfer matrices.
\end{abstract}

Keywords - Boundary Element Method, vector Boundary Element Method, Cortical Potential, Cortical Current Density, Inward Continuation.

\section{INTRODUCTION}

The estimation of the cortical potential distribution from that of the scalp is an inverse problem. The solution of this problem is unique, provided that there are no sources located between the cortical and scalp surface [1]. In order to solve this problem numerically, a relation between the potential distribution on the cortical and scalp surface has to be calculated. Therefore, it is required that node points are chosen at each surface. Subsequently, the relations that link the potential value at the node points of the cortex with those of the scalp can be put into a matrix. Customarily, a transfer matrix is found that is ill conditioned. Hence, the inverse solution is sensitive to small changes in the data. The condition number of a matrix indicates how ill conditioned a matrix is and is defined as the ratio of its largest and smallest singular value. A large condition number indicates that the matrix is ill conditioned.

In this paper two different transfer matrices will be studied. The first matrix relates the cortical potential to the potential at the scalp and is commonly used. The second one relates the current density at the cortex to the current density at the scalp and is proposed by Riera et al. [2]. The current density is directly linked to the potential by Ohm's law. Both types of matrices will be used in forward and inverse calculations using the boundary element method (BEM), where the contribution of the Ohmic current in the volume conductor is described by an equivalent contribution of secondary sources at the surfaces of the various compartments. In order to discriminate between the two types of methods, the method that is based on the relation between the potential distributions is called BEM and the one based on the current density (a vector) vBEM.

\section{Methods}

The relation between the distributions at the cortex and the scalp is derived for a model consisting of two compartments, i.e. the skull and the scalp. The BEM expression is given by equation (1) (see page 2 of this paper), $S_{1}, S_{2}$ and $S_{3}$ denote the cortical surface, the outer surface of the skull, and the scalp surface, respectively; $\sigma_{12}$ is the conductivity of the skull and $\sigma_{23}$ that of the scalp; $g=\frac{1}{\left|\vec{r}-\vec{r}^{*}\right|}$. Equation (1) is not ordinarily used. Instead of a primary source (usually a current dipole), each element $d S$ of the cortical surface $S_{1}$ is assumed to carry an elementary current $-\sigma_{12} \nabla \varphi \cdot \vec{n} d S$.

When the current density is used instead of the potential, the expression is given in equation (2). A vector with subscript $\perp$ or $/ /$ refers to a vector orientated normal or tangential to the surface. The current density vector is discontinuous at the interfaces. Therefore, the current density at the interfaces is taken as the limit approaching this surface from the inside or the outside. In expression (2), the current density vector of surface 3 is approached from the inside, and the current density vectors of surfaces 1 and 2 are approached from the outside.

For the numerical calculations the surfaces are divided into triangles. For the solution of equation (1), the potential is calculated at each node and a linear interpolation over each triangle is taken. For the solution of equation (2), the current density at each triangle is taken to be constant and is assumed to be equal to the current density that is calculated at the center of gravity of the triangle. As the current density is proportional to the gradient of the potential, the approximation that the current density is constant over a triangle corresponds to the approximation that the potential is a linear function over the triangle.

Subsequently, equation (1) is expressed by the following linear equations:

$$
\begin{aligned}
& \vec{\varphi}_{1}=\mathbf{M}_{1} \vec{\varphi}_{1}+\mathbf{M}_{2} \vec{\varphi}_{2}+\mathbf{M}_{3} \vec{\varphi}_{3}+\mathbf{M}_{4} \vec{J}_{1 \perp} \\
& \vec{\varphi}_{2}=\mathbf{M}_{5} \vec{\varphi}_{1}+\mathbf{M}_{6} \vec{\varphi}_{2}+\mathbf{M}_{7} \vec{\varphi}_{3}+\mathbf{M}_{8} \vec{J}_{1 \perp} \\
& \vec{\varphi}_{3}=\mathbf{M}_{9} \vec{\varphi}_{1}+\mathbf{M}_{10} \vec{\varphi}_{2}+\mathbf{M}_{11} \vec{\varphi}_{3}+\mathbf{M}_{12} \vec{J}_{1 \perp}
\end{aligned},
$$

where $\vec{\varphi}_{1}, \vec{\varphi}_{2}$ and $\vec{\varphi}_{3}$ are representing the potential values at the nodes of the cortical, outer skull and scalp surface, respectively and $\vec{J}_{1 \perp}$ is representing the normal current density at the nodes of the cortical surface. After elimination of the potential at the skull and the normal current 


$$
\begin{gathered}
4 \pi \varphi\left(\vec{r}^{*}\right)=-\oiint_{S_{3}} \varphi \nabla g \cdot \vec{n} d S-\frac{\sigma_{12}-\sigma_{23}}{\sigma_{23}} \oiint_{S_{2}} \varphi \nabla g \cdot \vec{n} d S-\frac{\sigma_{12}}{\sigma_{23}} \oiint_{S_{1}} \varphi \nabla g \cdot \vec{n} d S+\frac{\sigma_{12}}{\sigma_{23}} \oiint_{S_{1}} g \nabla \varphi \cdot \vec{n} d S \\
4 \pi J\left(\vec{r}^{*}\right)=-\oiint_{S_{3}} \nabla g \times\left(\vec{J}_{/ /} \times \vec{n}\right) d S-\frac{\sigma_{12}-\sigma_{23}}{\sigma_{23}} \oiint_{S_{2}} \nabla g \times\left(\vec{J}_{/ /} \times \vec{n}\right) d S-\oiint_{S_{1}} \nabla g\left(\vec{J}_{\perp} \cdot \vec{n}\right) d S+\oiint_{S_{1}} \nabla g \times\left(\vec{J}_{/ /} \times \vec{n}\right) d S
\end{gathered}
$$

where $g=\frac{1}{\left|\vec{r}-\vec{r}^{*}\right|}$ and $\vec{J}=\vec{J}_{/ /}+\vec{J}_{\perp}$.

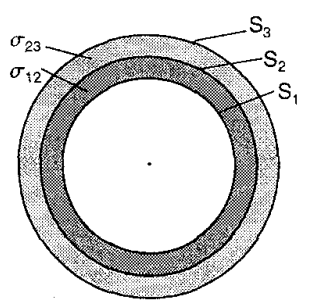

A Schematic model

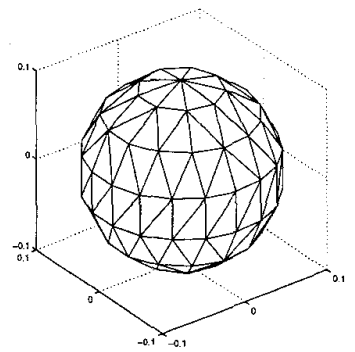

B 88 nodes, 172 triangles
Fig. 1. A $S_{1}, S_{2}$ and $S_{3}$ correspond to the surfaces of the cortex, the outside of the skull, and the scalp; $\sigma_{12}$ is the conductivity of the skull and $\sigma_{23}$ that of the scalp. $\mathbf{B}$ shows a triangulated surface with 88 nodes and 172 triangles.

density at the cortex from these linear relations, the following expression between the cortical potential and the scalp potential remains [3]:

$$
\vec{\varphi}_{3}=\mathbf{A}_{\mathbf{p}} \cdot \vec{\varphi}_{1}
$$

where $\mathbf{A}_{\mathbf{p}}$ is the desired transfer matrix. This matrix has dimension $N_{3} \times N_{1}$ where $N_{3}$ and $N_{1}$ are the numbers of nodes of both surfaces.

The same recipe is used for the numerical solution of equation (2). After elimination of the normal currents and the current densities at the interface between the skull and scalp, the transfer matrix reads:

$$
\vec{J}_{3 / /}=\mathbf{A}_{\mathbf{j}} \cdot \vec{J}_{1 / /}
$$

where $\vec{J}_{3 / /}$ and $\vec{J}_{1 / /}$ represent the tangential components of the current density at the triangles on the scalp and cortical surface, respectively. The transfer matrix $\mathbf{A}_{\mathbf{j}}$ has a dimension of $2 M_{3} \times 2 M_{1}$, where $M_{1}$ and $M_{3}$ are the number of triangles of both surfaces. Since the number of triangles is approximately twice as large as the number of nodes, matrix $\mathbf{A}_{\mathbf{j}}$ has sixteen times as much elements as matrix $\mathbf{A}_{\mathrm{p}}$.

To compare both transfer matrices, they are calculated for a model consisting of three spherical surfaces with radii of $0.08,0.09$ and $0.1 \mathrm{~m}$. As in this case analytical expressions for the potential and the current density are available, the accuracy of the BEM and vBEM can be tested. Two levels of discretizations are used, one having 88 nodes (172 triangles) and one having 203 nodes (402 triangles) at each interface (see Fig. 1). Two different ratios between the scalp and skull conductivities are used, namely $2: 1$ and
$80: 1$. The former ratio is the one Riera [4] used, the latter has a more realistic value. The dipole is located at the $\mathrm{z}$-axis and has an orientation with an angle of 45 degrees with the z-axis. Its eccentricity is varied. In the forward calculations, the distribution of the potential or the tangential components of the current density at the cortical surface are supposed to be known. For these distributions those are taken that would have been generated by a current dipole within the brain compartment of our model and that are calculated using an analytical expression. Starting from such a distribution, the scalp potential is calculated using the BEM and the current density at the scalp is calculated using the vBEM. Both results are compared with the analytical solution. The differences found are expressed by the RDM, $\mathrm{RDM}^{*}$ and the MAG factor [5]:

$$
\operatorname{RDM}_{\varphi}=\sqrt{\frac{\sum_{i=1}^{N}\left(\varphi_{i, c a l c}-\varphi_{i, a n}\right)^{2}}{\sum_{i=1}^{N}\left(\varphi_{i, a n}\right)^{2}}}
$$

$$
\operatorname{RDM}_{\varphi}^{*}=\sqrt{\sum_{i=1}^{N}\left(\frac{\varphi_{i, c a l c}}{\sqrt{\sum_{j=1}^{N}\left(\varphi_{j, c a l c}\right)^{2}}}-\frac{\varphi_{i, a n}}{\sqrt{\sum_{j=1}^{N}\left(\varphi_{j, a n}\right)^{2}}}\right)^{2}}
$$

$$
\begin{gathered}
\operatorname{MAG}_{\varphi}=\sqrt{\frac{\sum_{i=1}^{N}\left(\varphi_{i, c a l c}\right)^{2}}{\sum_{i=1}^{N}\left(\varphi_{i, a n}\right)^{2}}}, \\
\mathrm{RDM}_{J}=\sqrt{\frac{\sum_{k=1}^{M}\left(\left\|\vec{J}_{\| / k, \text { calc }}\right\|-\left\|\vec{J}_{/ / k, a n}\right\|\right)^{2}}{\sum_{k=1}^{M}\left(\left\|\vec{J}_{/ / k, a n}\right\|\right)^{2}}},
\end{gathered}
$$

$\mathrm{RDM}_{\mathrm{J}}^{*}=$

$$
\begin{gathered}
\sqrt{\sum_{k=1}^{M}\left(\frac{\left\|\vec{J}_{\| k, \text { calc }}\right\|}{\sqrt{\sum_{l=1}^{M}\left(\left\|\vec{J}_{\| l, c a l c}\right\|\right)^{2}}}-\frac{\left\|\vec{J}_{\| k, a n}\right\|}{\sqrt{\sum_{l=1}^{M}\left(\left\|\vec{J}_{/ l, a n}\right\|\right)^{2}}}\right)^{2}} \\
\mathrm{MAG}_{\mathrm{J}}=\sqrt{\frac{\sum_{k=1}^{M}\left(\left\|\vec{J}_{/ / k, \text { calc }}\right\|\right)^{2}}{\sum_{k=1}^{M}\left(\left\|\vec{J}_{/ / k, a n}\right\|\right)^{2}}}
\end{gathered}
$$

where $\varphi_{i, c a l c}$ is the calculated potential in node $i$ and $\varphi_{i, a n}$ is the analytical potential in node $i ;\left\|\vec{J}_{/ / k, \text { calc }}\right\|$ is the norm of the tangential components of the calculated current density at triangle $k$ and $\left\|\vec{J}_{/ / k, a n}\right\|$ is the norm of the tangential components of the analytical current density at triangle $k$. 
In the inverse calculations, the distribution at the scalp is supposed to be known. From this distribution, the distribution on the cortical surface is calculated. Hence, the left hand sides of equation (4) and (5) are known. The distribution at the cortex $\overrightarrow{\varphi_{1}}$ is using calculated using [6]:

$$
\vec{\varphi}_{1}=\mathbf{A}_{\mathrm{p}}^{-1} \cdot \vec{\varphi}_{3} .
$$

A similar inverse relation exists between the tangential components of the current density on the cortical and that on the scalp surface. As potential distribution at the scalp surface $\vec{\varphi}_{3}$, the potential distribution is taken that is generated by a current dipole positioned at the $\mathrm{z}$-axis $(\mathrm{z}=0.04 \mathrm{~m})$ having an angle of 45 degrees with the $z$-axis. Subsequently, different amounts of white noise are added to these potential values at the nodes of the scalp surface. The simulation is repeated 100 times for each noise level. For each triangle, the tangential components of the current density are calculated by taking the derivative of the linear function that describes the noisy potential on the triangle. The inverse solutions of the BEM and $v B E M$ are compared with analytical solutions. Again, the RDM, RDM* and MAG are used for the quantification of the differences found for the various noise levels.

\section{RESULTS}

The RDM, RDM* and MAG values for both the BEM and $\mathrm{vBEM}$ for a dipole at different eccentricities are shown in Fig. 2. The RDM and RDM* values increase with the eccentricity of the dipole. The MAG values deviate more and more from the desired value of 1 as the eccentricity of the dipole increases. For all four cases (two triangulations and two ratios of the conductivity), the results obtained with the BEM are more accurate than the results for the vBEM. For the conductivity ratio $80: 1$, the differences are larger than for the conductivity ratio of $2: 1$.

Table I lists the values of the condition number for the vBEM. The condition number increases with the conductivity ratio of scalp to skull. For the model with 88 nodes per interface and a conductivity ratio of $2: 1$, the transfer matrix of the BEM is the better-conditioned one. However, for the ratio $80: 1$, the condition number of the transfer matrix calculated using vBEM approach is lower than the condition number of the potential matrix. For the second model with 203 nodes per surface, the transfer matrix based on the BEM is the better-conditioned one. The condition number of the vBEM transfer matrix is for both conductivity ratios higher than the condition number of the BEM matrix.

The results of the inverse calculations with noise added to the potential distribution at the scalp are shown in Fig. 3. These values are obtained using the model consisting of 88 node points and a conductivity ratio of $80: 1$. From these figures, it can be seen that the error made us-
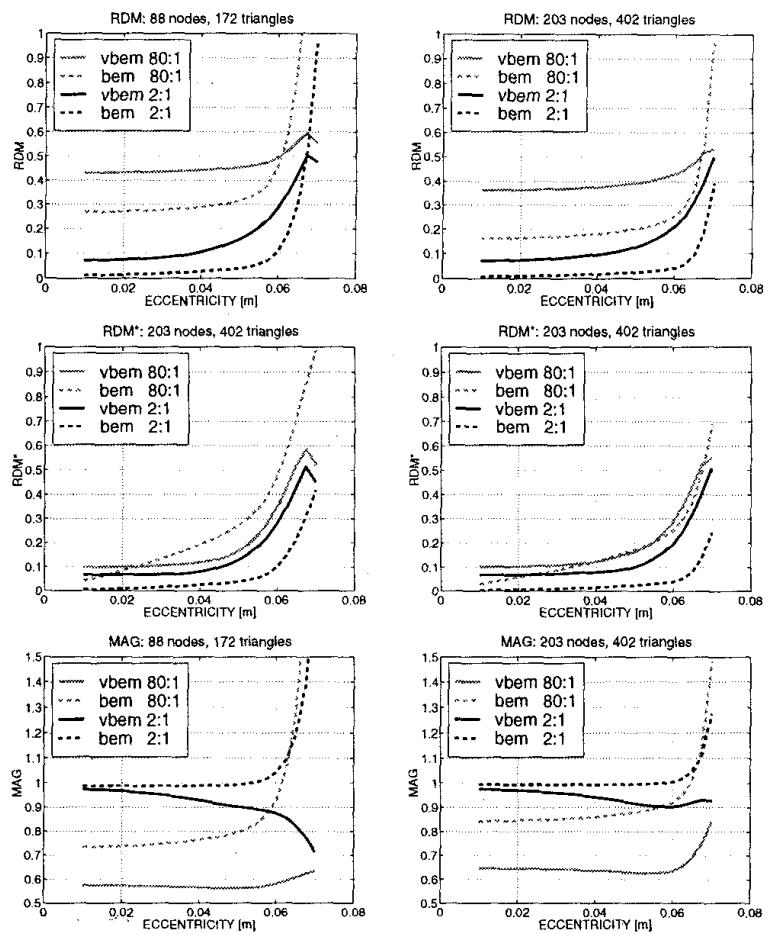

Fig. 2. Forward calculations: RDM (top row), RDM* (middle row) and MAG (bottom row) values for a dipole at different eccentricities on the z-axes. The left column shows the results for the triangulation with 88 nodes and 172 triangles per surface. The right column shows the results for the second model, each surface consisted of 203 nodes and 402 triangles. Solid lines represent the results for the vBEM; dashed lines the results for the BEM. Two different conductivity ratios were tested, $2: 1$ and $80: 1$.

TABLE I

Condition number of the (v)BEM transfer matrices for different ratios of the conductivity and the two different triangulations.

\begin{tabular}{cccc} 
Approach & $\begin{array}{c}\text { Model } \\
\text { (nodes) }\end{array}$ & $\begin{array}{c}\text { Conductivity ratio scalp to skull } \\
8: 1\end{array}$ \\
\hline BEM & 88 & 11.2 & $80: 1$ \\
vBEM & 88 & 37.2 & 336.8 \\
\hline BEM & 203 & 53.3 & 176.1 \\
vBEM & 203 & 326.9 & 1636 \\
\hline
\end{tabular}

ing the vBEM for inverse calculations is significantly larger than when the BEM is used.

\section{Discussion \& CONClUSiON}

A numerical relation between the cortical and scalp distribution of the tangential components of the current density is derived. The condition number of the transfer matrix gives an indication how ill posed the inverse problem is. The condition number of the transfer matrix found for the $\mathrm{VBEM}$ is not lower than the condition number found for the BEM. Hence, it cannot be stated that the trans- 

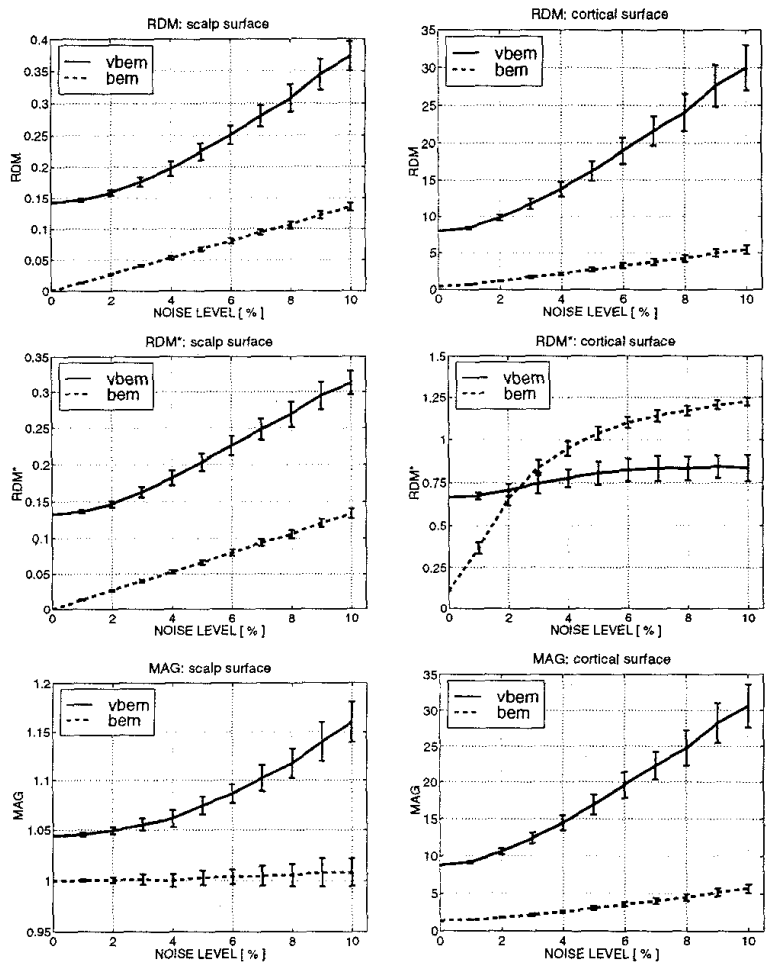

Fig. 3. Inverse calculations: mean RDM (top row), RDM* (middle row) and MAG (bottom row) factors for different amounts of white noise. The left column shows the results for the distributions on the scalp surface. The right column shows the results for the cortical distributions. Solid lines represent the error for the vBEM, dashed lines the results for the BEM. The results are shown for the model with 88 nodes and 172 triangles per interface and the conductivity ratio of $80: 1$. The standard devation of the RDM, RDM* and MAG are indicated with the errorbar.

fer matrix based on the vBEM is more suitable for inverse calculations. This conclusion is in contrast with the conclusions made by Riera et al. [2], [4], who obtained for their problem better results when they used the vBEM.

The normal and the tangential components at the cortical surface are related. If the forward solution would be based on all these components, the solution would probably be more accurate. In case of inverse calculations, adding the normal component will not give an additional advantage, as it only introduces an extra variable that has to be determined.

Generally, the potential distribution on the scalp is known from measurements. The tangential components of the current density have to be calculated from these measurements by taking the derivative of the potential distribution. As can be deduced from Fig. 3, this step results in a substantial error, as in the noise free case the RDM between the analytical and the calculated tangential components of the current density is 0.14 .

The $\mathrm{vBEM}$ is nothing more than the differentiation of the BEM. Apart from the reference potential, which is lost during the differentiation, there should only be a numerical advantage. Therefore, most problems of the BEM will remain.

It is doubtful whether the $\mathrm{VBEM}$ is suitable for inverse calculations. The vBEM is sensitive to potential distributions that are contaminated with noise. Furthermore, the $\mathrm{vBEM}$ requires more computational effort. The vBEM transfer matrix is approximately 16 times as large compared to the transfer matrix based on the BEM. Taking these disadvantages into account, it is not recommendable to solve this kind of inverse problem with the vBEM.

\section{REFERENCES}

[1] Y. Yamashita, "Theoretical studies on the inverse problem in electrocardiography and the uniqueness of the solution", IEEE Trans. Biomed. Eng., vol. 29, no. 11, pp. 719-725, 1982.

[2] J. Riera, M. Fuentes, E. Aubert, and D. Díaz, "Solving the forward problem: Spherical vs. realistic electric leadfield", Biomedizinische Technik, vol. 42 (sup 1), pp. 223-226, 1997.

[3] A. van Oosterom and T.F. Oostendorp, "On computing pericardial potentials and current densities in inverse electrocardiography", J. Electrocardiology, vol. 25, no. sup, pp. 102-106, 1992.

[4] J.J. Riera and M.E. Fuentes, "Electric lead field for a piece-wise homogeneous volume conductor model of the head", IEEE Trans. Biomed. Eng., accepted for publication, 1998.

[5] J. Meijs, M. Peters, H. Boom, and F. Lopes da Silva, "Relative influence of model assumptions and measurement procedures in the analysis of the meg", Med. \& Biol. Eng. \& Comput., vol. 26, pp. 136-142, 1988.

[6] G. Golub and C. van Loan, Matrix computations, John Hopkins Univ. Press, Maryland, $2^{\text {nd }}$ edition, 1989. 